\title{
Immuncheckpointinhibition bei therapierefraktären Kopf-Hals-Tumoren
}

\section{Der PD-L1-Signalweg spielt auch bei Kopf-Hals-Plattenepithelkarzinomen (HNSCC) eine Rolle. Nun wurde die Wirksamkeit des gegen PD-1 gerichteten Antikörpers Pembrolizumab in dieser Indikation untersucht.}

1 a atienten mit rezidivierten/metastasierten (R/R) HNSCC, die gegenüber Platin und Cetuximab refraktär sind, haben eine schlechte Prognose. Für sie gibt es derzeit keine zugelassenen Therapieoption. In der Phase-II-Studie KEYNOTE-055 wurde nun der Anti-PD1Antikörper Pembrolizumab in dieser Population untersucht.

171 Patienten mit Krankheitsprogression innerhalb von 6 Monaten nach Ende der Therapie mit Platin und Cetuximab erhielten Pembrolizumab $200 \mathrm{mg}$ alle 3 Wochen bis zur Progression. 75\% der Patienten hatten bereits $\geq 2$ Vortherapien erhalten, $82 \%$ waren PD-L1-positiv, $22 \%$ HPV-positiv. Primäre Endpunkte waren Ansprechen und Sicherheit.

Bei $64 \%$ der Patienten kam es zu therapiebedingten unerwünschten Ereignissen, bei $15 \%$ vom Grad $\geq 3$. Am häufigsten waren Fatigue, Hypothyreose, Übelkeit, AST-Anstieg und Diarrhö.
7 Patienten (4\%) brachen die Therapie ab, einer starb an einer therapiebedingten Pneumonitis.

Die Gesamtansprechrate auf die Therapie mit Pembrolizumab lag bei $16 \%$, die

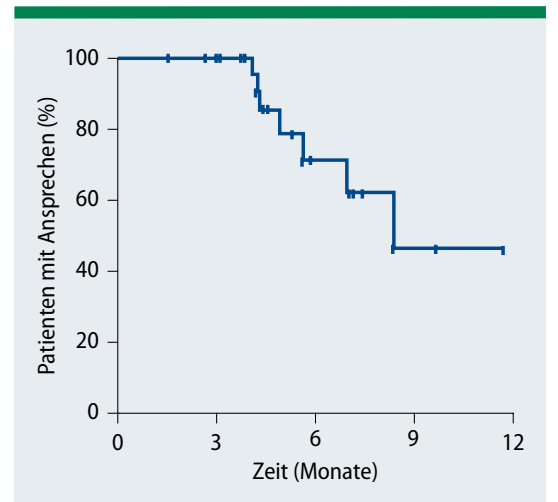

Abb. 1: Bei Patienten mit bestätigtem Ansprechen nach RECIST-Kriterien (v1.1) hielt dieses zum Teil relativ lange an. mediane Dauer des Ansprechens bei 8 Monaten (Abb. 1). Ein Patient erreichte eine Komplettremission. Bei 19\% der Patienten blieb die Erkrankung stabil, bei $51 \%$ kam es zur Progression. Die Ansprechraten waren unabhängig vom HPV-Status. Patienten mit höherer PD-L1-Expression erreichten höhere Ansprechraten. Das progressionsfreie Überleben betrug median 2,1, das Gesamtüberleben 8 Monate. Auch beim Überleben gab es keine Unterschiede hinsichtlich des HPV-Status, ebensowenig hinsichtlich der PD-L1-Expression. Damit bleibt der therapeutische Nutzen von Pembrolizumab nicht auf Patienten mit PD-L1-Expression beschränkt.

Fazit: Pembrolizumab hatte beim rezidivierten/metastasierten, vorbehandelten HNSCC eine klinisch relevante AntiTumoraktivität und ein akzeptables Sicherheitsprofil. Judith Neumaier

Bauml J et al. Pembrolizumab for Platinum- and Cetuximab-Refractory Head and Neck Cancer: Results From a Single-Arm, Phase II Study. J Clin Oncol. 2017;35(14):1542-9.

\section{Lebensqualität unter der Therapie bei Kopf-Hals-Tumoren}

\begin{abstract}
Aktuelle Studien bei lokoregionär fortgeschrittenen Kopf-Hals-Plattenepithelkarzinomen (LA-HNSCC) zielen vor allem auf eine mindestens gleichwertige Wirksamkeit bei besserem Funktionserhalt ab. Daten zur Lebensqualität sind aber immer noch selten.
\end{abstract}

n einer kanadischen Phase-III-Studie wurden jetzt Lebensqualitätsparameter zwischen zwei Therapien bei 320 Patienten mit nicht metastasierten, nodal positiven Tumoren jedes Stadiums oder T34-Tumoren ohne Lymphknotenmetastasen (81\% Oropharynx-Tumoren) untersucht. Randomisiert haten die Patienten entweder eine Radiochemotherapie (Standardarm A: 70 Gy in 35-Fraktionen über 7 Wochen plus Cisplatin) oder eine
Radiotherapie (Interventionsgruppe B: Radiotherapie 70 Gy in 35-Fraktionen über 6 Wochen) ergänzt um Panitumumab. Die Lebensqualität wurde zu Studienbeginn und nach Ende der Bestrahlung sowie 2, 4, 6, 12, 24 und $36 \mathrm{Mo}$ nate danach mit „Functional Assessment of Cancer Therapy Head and Neck“ (FACT-H\&N), „MD Anderson Dysphagia Index“ (MDADI) und „SWAL-QOL“ erhoben.

$\mathrm{Zu}$ Beginn unterschieden sich die Lebensqualitätswerte zwischen den Armen nicht. Die FACT-H\&N-Scores unterschieden sich entgegen der Erwartung aber auch nach einem Jahr nicht $(p=0,194)$. Nur in der letzten Bestrahlungswoche gab es in einigen Subskalen signifikante Unterschiede, aber zugunsten von Arm A, so bei der physischen FACT-Subskala $(p=0,049)$, bei der phy- sischen Subskala des MDADI $(\mathrm{p}=0,045)$ und den SWAL-QOL-Subskalen Mahlzeitendauer $(\mathrm{p}=0,02)$, Esswunsch $(\mathrm{p}=0,031)$ und psychische Gesundheit $(\mathrm{p}=0,009)$.

Nach Therapieende fanden sich keine klinisch bedeutsamen Unterschiede mehr.

Fazit: Panitumumab zusätzlich zur Strahlentherapie kann die Lebensqualität und die Schluckfunktion gegenüber einer Cisplatin-unterstützten Strahlentherapie nicht verbessern. Friederike Klein

Ringash J et al. Quality of life and swallowing with standard chemoradiotherapy versus accelerated radiotherapy and panitumumab in locoregionally advanced carcinoma of the head and neck: A phase III randomised trial from the Canadian Cancer Trials Group (HN.6). Eur J Cancer 2017;72:192-9. 